\title{
Atomic Observation of Phase Transformation from Spinel to Rock Salt in Lithium Manganese Oxide
}

\author{
Peng Gao ${ }^{1}$, Ryo Ishikawa ${ }^{1}$, Eita Tochigi ${ }^{1}$, Akihito Kumamoto ${ }^{1}$, Naoya Shibata ${ }^{1}$, Yuichi Ikuhara ${ }^{1}$ \\ ${ }^{1}$ Institute of Engineering Innovation, School of Engineering, The University of Tokyo, Tokyo 113-8656, \\ Japan
}

Solid-state phase transformation are ubiquitous and of critical importance to numerous technological applications, such as inorganic ceramics, alloy and energy conversion and storage. These transformation processes usually involve only local atomic displacements without adding/reducing any atoms or longrange atom migration. For example, in perovskite ferroelectrics the oxygen octahedral shift and/or rotation results in a first order phase transition-polarization switching [1]. Investigating the dynamic processes of phase transformation in solids is critical to gain insights to understanding the fundamental physical properties of functional materials and benefiting industrial technologies.

Here we show an atomic-scale observation of phase transformation from spinel to rock salt in lithium manganese oxide by using aberration corrected scanning transmission electron microscopy (STEM). Viewing along the [110] direction of spinel $\mathrm{LiMn}_{2} \mathrm{O}_{4}$ (space group $\mathrm{Fd} 3 \mathrm{~m}$ ), the $\mathrm{Li}$, $\mathrm{O}$ and $\mathrm{Mn}$ are well separated from each other (Fig. 1a). A 'diamond' configuration consists of eight Mn columns in which the Mn density at Mn1 column is twice that of the $\mathrm{Mn} 2$ columns. The $\mathrm{O}$ atoms in the $\mathrm{O} 1$ columns are slightly misaligned and lean towards Mn1 sites (Fig.1a) while the two Li columns are separated by $\sim 0.2$ $\mathrm{nm}$ at the center of diamond. In our experiments, scanning the electron probe over a thin edge region of oxygen deficient $\mathrm{LiMn}_{2} \mathrm{O}_{4-\mathrm{x}}(\mathrm{LMO})$ particle will drive the spinel structure convert into rock salt (Fig. 1a) via local atom migration without any mass loss which is evidenced by electron energy loss spectroscopy (EELS) in Fig.1b. Successive fast-scanned STEM frames are recorded to track this dynamic process.

During the phase transformation, the intensity of Mn1 sites in the high angle annular dark-field (HAADF, Z-contrast) image is significantly reduced (35\%) while the intensity at Mn3 columns gradually becomes brighter (in Fig. 1c), indicating Mn atoms migrate from Mn1 to Mn3 atomic sites. At $58 \mathrm{~s}$ in Fig.1c, the intensity between Mn1 and Mn2 columns is close because those columns have the same Mn density in the rock salt structure. Moreover we observe the anions shift during phase transformation. Simultaneously recorded sequential annular bright-field (ABF) images are shown in Fig. $1 \mathrm{~d}$. With the spinel structure, the $\mathrm{O} 1$ columns in the $\mathrm{ABF}$ image are not resolvable because the contrast of Mn1 columns is too intense and $\mathrm{O} 1$ columns are too close to Mn1. Once the structure has transformed into rock salt, the intensity of Mn1 is reduced significantly as half of Mn has been replaced by lithium. Furthermore, in the rock salt structure the O1 columns shift always from the Mn1 and are aligned perfectly and hence appear distinguishable in the ABF image as indicated by the arrows in Fig. 1d.

Based on these observations, the phase transformations can be summarized as two step reactions: 1) tetrahedrally coordinated Li migrates to octahedral sites; 2) Mn migrates from one octahedral site to another. The detailed changes in composition, occupancy and electronic structures of both heavy and light atomic columns determined by STEM imaging (HAADF and ABF) and EELS, lead to useful insights into solid-state phase transformations and will bring beneficial technological solutions to a variety of applications such as the lithium ion batteries [2]. 
References:

[1] P.Gao et al, Nature Communications 2 (2011) 591.

[2] The authors gratefully acknowledge the financial support through a Grant-in-Aid for Scientific

Research on Innovative Areas "Nano Informatics" (Grant No. 25106003) from Japan Society for the Promotion of Science (JSPS), and "Nanotechnology Platform" (Project No. 12024046) from the Ministry of Education, Culture, Sports, Science and Technology in Japan (MEXT).

a

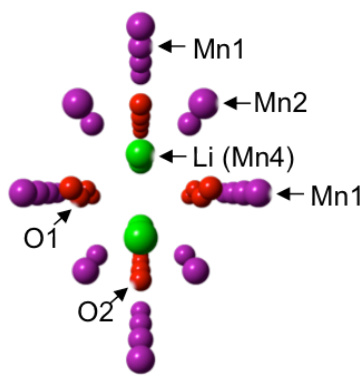

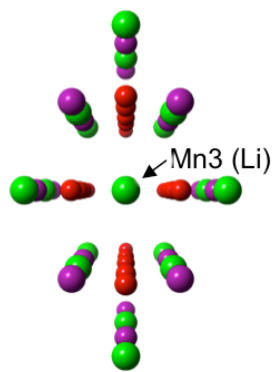

b
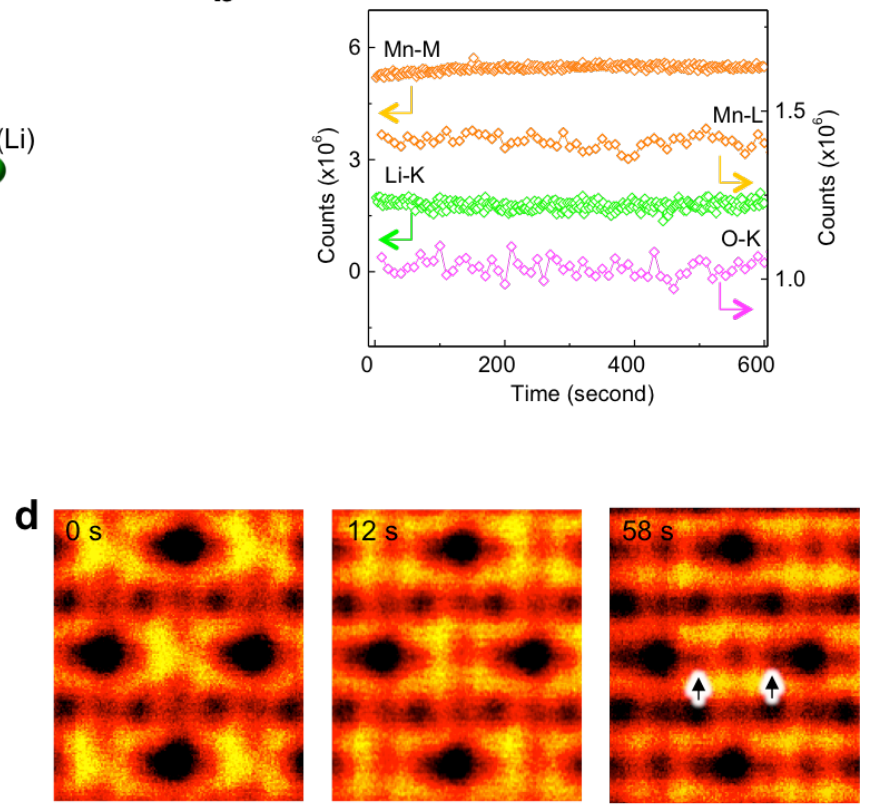

$0.583 \mathrm{~nm}$
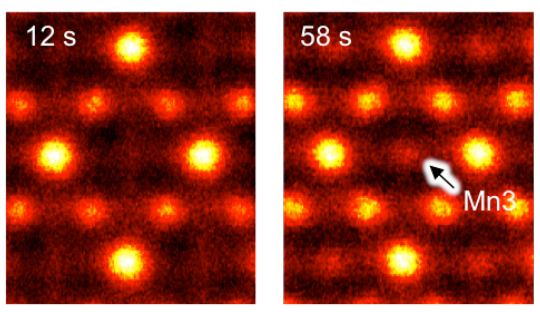

Figure 1. (a) A perspective view of spinel $\mathrm{LiMn}_{2} \mathrm{O}_{4}$ along [110] direction and rock salt $\mathrm{LiMnO}_{2}$. Green: Li. Purple: Mn. Red: O. In the spinel phase, the atom density of Mn1 columns is twice of Mn2 columns. In the rock salt phase, the atom densities of Mn1, Mn2 and Mn3 sites are the same. Li site is unoccupied. (b) Intensities of simultaneously recorded Li-K and Mn-M edges, and simultaneously recorded O-K and $\mathrm{Mn}-\mathrm{M}$ are plotted as a function of time showing no atom loss during phase transformation. Selected HAADF-STEM (c) and ABF-STEM (d) continuous images showing the phase transition from oxygen deficient spinel to lithium deficient rock salt in $\mathrm{LiMn}_{2} \mathrm{O}_{4-\mathrm{x}}$ (x denotes oxygen vacancies). Commercial $\mathrm{LiMn}_{2} \mathrm{O}_{4}$ nanoparticles were annealed in $\mathrm{N}_{2}$ at $700^{\circ} \mathrm{C}$ to form oxygen deficient $\mathrm{LiMn}_{2} \mathrm{O}_{4-\mathrm{x}}$. In the STEM images with 'TIFF' format, the intensity at each pixel has been automatically normalized between 0 and 255 , the precise comparison in intensity is calculated from the original DM format. HAADF and ABF images are recorded simultaneously at $300 \mathrm{kV}$. The interval between frames is $\sim 2 \mathrm{~s}$. Each frame is 1024 by 1024 pixels in size. A post drift correction was applied to align the frames. Theses selected frames are unit-cell averaged from a region of 5 by 5 unit cells to reduce the noise. The arrows in (d) indicating the $\mathrm{O} 1$ sites in rock salt are resolvable from Mn1 sites contrasting to the pristine spinel structure. 